\title{
Papel moderador de los estilos parentales en la relación entre la impulsividad y el consumo de alcohol en una muestra de adolescentes españoles
}

\author{
Gloria Pérez de Albéniz-Garrote ${ }^{1}$, Laura Rubio-Rubio² y Begoña Medina-Gómez ${ }^{1}$ \\ ${ }^{1}$ Universidad de Burgos, Burgos, España \\ ${ }^{2}$ Universidad de Granada, Granada, España
}

The moderating role of parenting styles in the relationship between impulsivity and alcohol consumption in a sample of Spanish adolescents

\begin{abstract}
Alcohol consumption in adolescents is a complex problem that is often related to impulsive behavior. The objective of this study was to analyze the role of parenting styles in the relationship between impulsivity and alcohol consumption in a sample of 613 Spanish adolescents aged between 13 and 18 years $(M=15.76, D T=1.16)$ and enrolled in secondary education schools. The results revealed that neglect and negative evaluation of the mother as well as a controlling father were the variables related to alcohol consumption in male adolescents with high dysfunctional impulsive. In the case of female adolescents, support and stimulation, as well as a positive evaluation of the father were linked to lower consumption. This study presents evidence that the parenting style of the parents is related to alcohol consumption of children depending on the degree of dysfunctional impulsivity.
\end{abstract}

Keywords: Adolescents; impulsivity; alcohol; parenting styles.

Resumen: El consumo de alcohol en adolescentes es un problema complejo que suele aparecer acompañado de conductas impulsivas. El objetivo de este estudio fue analizar el papel de los estilos parentales en la relación entre la impulsividad y el consumo de alcohol en una muestra de 613 adolescentes españoles escolarizados en centros de educación secundaria, con edades comprendidas entre los 13 y 18 años $(M=15.76, D T=1.16)$. Los resultados indicaron que la negligencia y la evaluación negativa de la madre, así como el control del padre eran las variables relacionadas con el consumo de alcohol en varones con elevada impulsividad disfuncional. En las mujeres, el apoyo y estimulación, y la evaluación positiva del padre se vinculaban a un menor consumo. Este estudio presenta evidencia de que el estilo de crianza de los padres está relacionado con el consumo de alcohol de los hijos dependiendo del grado de impulsividad disfuncional de éstos últimos.

Palabras clave: Adolescentes; impulsividad; alcohol; estilos de crianza.

\section{Introducción}

Los comportamientos, actitudes y creencias que poseen los padres van a definir la manera que tienen de interactuar con sus hijos. Estas pautas de relación se pueden combinar de múltiples maneras y categorizarse en una serie de dimensiones que, relacionadas entre sí, dan

Recibido: 02 de junio 2017; aceptado: 29 de agosto 2017

Correspondencia: Gloria Pérez de Albéniz-Garrote, Facultad de Educación, Universidad de Burgos, C/ Villadiego s/n, 09001 Burgos, España. Correo-e: ggarrote@ubu.es lugar a distintos tipos de educación familiar que conforman los estilos parentales (Coloma, 1993).

Uno de los modelos teóricos clásicos, el propuesto por Baumrind (1966), establece tres estilos educativos paternos: el autoritario, el democrático y el indulgente. Posteriormente, esta teoría fue reformulada y se añadió el estilo negligente (Maccoby y Martin, 1983). Fundamentalmente, la clasificación de las prácticas educativas durante la crianza se ha organizado sobre dos dimensiones centrales: el afecto y el control. De esta manera, los padres con estilo educativo autoritario se caracterizan por ejercer un control férreo de la conducta del hijo, por 
valorar la obediencia y por tener diálogo y afecto explícito escasos. Por su lado, los padres con estilo democrático o autoritativo ponen el énfasis en la comunicación bidireccional y el afecto, pero con control del comportamiento del hijo. Los padres con estilo educativo permisivo o indulgente basan su crianza en potenciar la autonomía del menor, con control laxo y alto afecto. Por último, los padres con estilo educativo negligente se caracterizan por la baja implicación en todos los aspectos de la crianza.

Han sido muchas las investigaciones en las que se ha analizado qué estilo de crianza proporciona un mejor ajuste psicosocial de los niños y adolescentes, reconociéndose en la mayoría de estudios unos mayores beneficios del estilo democrático. Sin embargo, aunque existe consenso sobre la importancia de la variable afecto para el desarrollo armónico de los menores, el acuerdo es menor en lo que se refiere a la variable control. De hecho, en los últimos años son varias las voces en nuestro país que consideran que puede tener ventajas el estilo indulgente, basado en el uso de la aceptación, el afecto, la utilización del diálogo, el razonamiento con los hijos y una baja severidad (Fuentes, Alarcón, Gracia, y García, 2015; Pérez Alonso-Geta, 2012).

Resulta también interesante la propuesta realizada por Schaefer (1965) y la posterior adaptación que realizan sobre ella Samper, Cortés, Mestre, Nácher, y Tur (2006), que plantean la existencia de cuatro dimensiones centrales: control, amor, autonomía y hostilidad. La combinación de estas dimensiones se traduce en ocho estilos de crianza. Algunos de esos estilos serían positivos o más adaptativos, como es el caso de los basados en el apoyo y la estimulación que ofrecen los padres a la toma de decisiones de sus hijos, el grado de permisividad o autonomía que están dispuestos a darles, o las evaluaciones positivas que realizan de sus comportamientos. Otros son desadaptativos como la hostilidad, entendida como exceso de rigor y utilización del castigo, la sobreprotección, la negligencia (descuido e ignorancia de lo que hace el hijo), las evaluaciones negativas (concebidas como quejas, control a través de la culpa o irritabilidad) o la desaprobación de las conductas del hijo. Por lo tanto, el estilo parental es un constructo multidimensional, por lo que en la práctica puede resultar difícil encajar a los padres en una u otra categoría. A este respecto, se han elaborado instrumentos de autoinforme con la finalidad de medir componentes específicos de los comportamientos de los progenitores durante la crianza.

Por otro lado, los comportamientos parentales no son estáticos e invariables, sino que pueden ir cambiando y ajustándose a lo largo del tiempo, de acuerdo con variables como el temperamento, la edad o el sexo del hijo.
Cabría pensar que con la llegada de la adolescencia la familia tiene menos influencia sobre el desarrollo y el ajuste del adolescente, pero en la mayoría de los casos las relaciones siguen siendo estrechas y afectuosas y los padres continúan siendo un factor esencial para favorecer el desarrollo de los hijos también en esta etapa (Hernando, Oliva, y Pertegal, 2012). No obstante, es un momento evolutivo de cambio que puede influir de manera significativa en la manera de ejercer la parentalidad. Del mismo modo, el estilo de relación que manejen los padres puede ser decisivo en los comportamientos que manifieste el adolescente (Collins y Laursen, 2004).

Asimismo, se ha estudiado el papel de los estilos parentales en el establecimiento de buenos hábitos para la salud del adolescente, por ejemplo, en relación al consumo de alcohol. De esta manera, la falta de vínculos familiares, los conflictos entre los padres, la negligencia, la ambigüedad en las normas, el exceso de protección o de permisividad con los hijos, o una organización familiar rígida han mostrado tener una influencia negativa en el consumo de sustancias tóxicas por parte de los hijos. En cambio, las buenas relaciones familiares, la comunicación bidireccional entre el adolescente y sus padres, así como un adecuado apego familiar han mostrado ser factores protectores en relación con el consumo de alcohol en estas edades (Alfonso, Huedo-Medina, y Espada, 2009; Becoña et al., 2012; Kenney, Lac, Grimaldi, y LaBrie, 2015; Martínez, Fuentes, García, y Madrid, 2013; Martínez-Loredo et al., 2016; Pons y Berjano, 1997).

Actualmente, el consumo de sustancias tóxicas sigue siendo una de las preocupaciones fundamentales en relación con la salud en la etapa de la adolescencia y un serio problema de salud pública. Aunque el último informe del Plan Nacional sobre Drogas (ESTUDES, 2016) muestra que la prevalencia de consumo de alcohol ha disminuido ligeramente en la adolescencia, los porcentajes siguen siendo elevados: el $76.8 \%$ de los adolescentes entre los 14-18 años ha consumido alcohol en el último año, y el $68.2 \%$ en el último mes. Por otro lado, en un estudio realizado por Ballester y Gil (2009), el 89\% de los adolescentes encuestados indicó que el consumo de alcohol entre sus amigos era algo habitual. La ingesta sigue produciéndose en un contexto de ocio, socialización e iniciación a la adolescencia, muy ligado al deseo de experimentar y a la búsqueda de vivencias novedosas, y se produce frecuentemente durante el fin de semana y en sesiones de pocas horas de duración, lo que se ha denominado patrón de binge drinking o atracón (Golpe, Isorna, Barreiro, Braña, y Rial, 2017; Petit, Maurage, Kornreich, Verbanck, y Campanella, 2014).

Diversas investigaciones muestran que tener contacto con el alcohol a temprana edad puede afectar tanto al de- 
sarrollo físico, como al conductual y al cognitivo (Carballo, García, Jáuregui, Marín, y Pérez-Jover, 2011), especialmente en relación al desarrollo de las áreas prefrontales del cerebro, involucradas en procesos de regulación, planificación y ejecución de la acción (López-Caneda et al., 2014; Pedrero y Ruiz, 2011). Además, es un factor de riesgo tanto para desarrollar patrones de uso abusivo de alcohol en la edad adulta como para desarrollar un consumo de otras sustancias (García-Moreno, Expósito, Sanhueza, y Angulo, 2008; Hernández-López et al., 2009), ya que el consumo de alcohol está presente en al menos en el 95\% de los policonsumos según el Observatorio Español de la Droga y las Toxicomanías (OEDT, 2015). Además, este patrón actual de ingesta tipo binge drinking o atracón, incrementa los riesgos para la salud tanto física como psíquica de los adolescentes (Luque, Gómez, Cortés, Espejo, y Giménez, 2014).

Sin embargo, no solo el estilo parental influye en el consumo de alcohol. Una de las variables personales relacionadas con este tipo de consumo en la adolescencia es la impulsividad, que podemos definir como un constructo multidimensional vinculado al control del comportamiento y de las emociones, y caracterizado por una pronunciada tendencia a ejecutar acciones sin una planificación adecuada (Echeburúa y De Corral, 2010). Esta variable aparece relacionada, generalmente, con dificultades para procesar la información y sostener la atención; dificultades que pueden ocasionar diversos problemas (Orozco-Cabal y Herin, 2008). Pero no toda conducta impulsiva tiene por qué ser problemática. En determinadas ocasiones, un estilo impulsivo de respuesta puede beneficiar al sujeto, sobre todo en tareas simples en las que se requiere una ejecución rápida en un tiempo breve. Por ello, algunos investigadores han propuesto la existencia de dos tipos de impulsividad: funcional y disfuncional. La impulsividad funcional hace referencia a un estilo rápido de procesamiento de la información que conduce a respuestas correctas y a un mejor manejo de los recursos atencionales. La impulsividad disfuncional, en cambio, está relacionada con la tendencia a tomar decisiones rápidas pero irreflexivas y poco planificadas, lo que puede derivar en interferencias en la comprensión de las instrucciones y en la planificación de las acciones a ejecutar, así como en dificultades para mantener la atención en el desarrollo de la acción (Pedrero, Ruiz, Rojo, Llanero, y Puerta, 2012).

En la etapa de la adolescencia los comportamientos impulsivos disfuncionales pueden hacer a los adolescentes más vulnerables a realizar conductas de riesgo para su salud como abusar del alcohol (Narváez y Caro, 2015). Al mismo tiempo, un contexto de crianza afectuoso y enriquecedor en el que se realicen actividades que fomenten la planificación y la toma de decisiones puede ayudar a la maduración de la corteza prefrontal del adolescente (Oliva y Antolín, 2010). En este sentido, los estilos parentales autoritarios se han mostrado como un factor de riesgo para el desarrollo de conductas impulsivas en los adolescentes mientras que los estilos parentales democráticos parecen ayudar a modelar los impulsos del adolescente (Iglesias y Romero, 2009; Raya, Herruzo, y Pino, 2008).

Por último, las investigaciones resaltan que la crianza paterna y materna se percibe diferencialmente por los hijos y que esta percepción parece estar claramente mediada por el sexo de los progenitores (Campano, González-Tornaría, y Massonnier, 2016; Tur, Mestre, Samper, y Malonda, 2012).

Teniendo en cuenta este marco de referencia, el presente estudio propone los siguientes objetivos: 1) Analizar el impacto de los estilos parentales y de la impulsividad de los hijos adolescentes sobre el consumo de alcohol de estos últimos; 2) Determinar si existe un papel moderador de los estilos de crianza parentales en el impacto de la impulsividad sobre el consumo de alcohol de los hijos; y 3) Analizar el papel moderador de los estilos parentales en función del género del progenitor.

\section{Método}

\section{Participantes}

En primer lugar, se realizó una reunión con responsables de la Dirección Provincial de Educación de Burgos (España) para informarles sobre el estudio, los objetivos y la finalidad del mismo. Se acordó incluir únicamente centros ubicados en la ciudad de Burgos para la realización del estudio. Posteriormente, la muestra se seleccionó empleando un muestreo aleatorio por conglomerados, siendo la unidad de muestreo los centros educativos. La Dirección Provincial informó a los directores de los centros sobre la realización del estudio. Se aseguró que el número de centros públicos o privados/concertados del estudio fuera representativo de la realidad observada en la ciudad a este respecto, por lo que fueron incluidos 5 centros públicos y 3 centros concertados. El único criterio de inclusión para que el adolescente pudiera participar en el estudio era estar escolarizado en alguno de los cursos incluidos en el estudio. No se establecieron criterios de exclusión.

En el estudio participaron un total de 613 alumnos escolarizados en centros de la ciudad de Burgos (España) con edades comprendidas entre los 13 y 18 años $(M$ $=15.76, D T=1.16)$, de los cuales el $48.9 \%$ eran varones y el $51.1 \%$ mujeres. El $23.1 \%$ cursaba $3^{\circ}$ de ESO, el $26.7 \% 4^{\circ}$ de ESO, el $27.6 \% 1^{\circ}$ de Bachillerato y el $22.3 \%$ 
$2^{\circ}$ de Bachillerato. El 86\% manifestó vivir con ambos padres, el $11 \%$ vivía únicamente con la madre, el $2 \%$ con el padre y un .6\% con otros familiares.

\section{Procedimiento}

En primer lugar, se enviaron cartas informativas a los directores de los centros seleccionados. Posteriormente, en una reunión presencial se informó al director/a de los objetivos y pasos de la investigación. A continuación, se solicitó el consentimiento informado a los alumnos de los centros. En el caso de los alumnos menores de edad, fue la dirección del centro la que solicitó el consentimiento informado de los padres o tutores legales de los alumnos. Todos los alumnos de los centros seleccionados accedieron a participar en el estudio.

El equipo investigador junto con el tutor del grupo facilitó el cuadernillo con los instrumentos de medida a los participantes. La duración aproximada de la aplicación de las pruebas fue de 20 minutos. Estas pruebas se aplicaron colectivamente en las respectivas aulas, y siempre se siguió el mismo orden en la aplicación de los instrumentos. Para controlar la deseabilidad social en las respuestas, se informó a los alumnos de la confidencialidad de los datos y de que las respuestas eran anónimas. Asimismo, una vez los alumnos comenzaban a rellenar los cuestionarios se pidió al tutor de cada clase que abandonara el aula.

\section{Instrumentos}

Inventario para Hijos/as del Comportamiento Parental (Child's Reports of Parental Behavior Inventory, CRPBI; Schaefer, 1965). Para este trabajo se empleó la adaptación española realizada por Samper, Cortés, Mestre, Nácher, y Tur (2006). Esta escala evalúa la percepción que tienen los hijos de los estilos parentales y la relación con su padre y con su madre. La aplicación del instrumento permite obtener dos puntuaciones diferentes en cada una de las dimensiones del cuestionario, una referente al padre y otra a la madre. Consta de 52 ítems de tipo Likert con tres opciones de respuesta $(1=$ nunca; $2=$ algunas veces; 3 = siempre). El análisis factorial realizado en la adaptación al castellano del instrumento muestra la existencia de ocho dimensiones o factores que explicaban un $42 \%$ en la varianza total para las madres y un $45.2 \%$ en el caso de los padres. Las dimensiones presentan ligeras variaciones en el número de ítems para padres y madres. Para ambos progenitores existen seis dimensiones comunes: apoyo y estimulación a la toma de decisiones, evaluación negativa, evaluación positiva, negligencia, permisividad y desaprobación. Además, en el caso de la madre las dos dimensiones restan- tes son hostilidad-rigidez y sobreprotección mientras que en el caso de los padres son sobreprotección-ignorancia y control. La adaptación española mostró propiedades psicométricas adecuadas con valores de fiabilidad de .57 para la madre (valores desde .30 hasta .85 según la subescala) y de .66 para el padre (valores que oscilaron desde .41 hasta .88 según la subescala) (Samper et al., 2006). Para el trabajo que se presenta los valores alfa de Cronbach fueron altos: en el caso de las escalas maternas fue de .76 (el valor más bajo fue de .49 para desaprobación y el más elevado .89 para apoyo y estimulación a la toma de decisiones), y de .78 para las escalas paternas (desde .30 para desaprobación, hasta .77 para apoyo y estimulación a la toma de decisiones).

Cuestionario de Impulsividad (Dickman's Impulsivity Inventory, DII; Dickman, 1990). Se utilizó la adaptación al castellano de Chico, Tous, Lorenzo-Seva, y Vigil-Colet (2003). El instrumento está formado por 23 ítems de respuesta dicotómica (Verdadero/Falso). Evalúa dos tipos de impulsividad: la impulsividad funcional (11 ítems), relacionada con el entusiasmo, riesgo, actividad y audacia, y la impulsividad disfuncional (12 ítems) relacionada con conductas desordenadas e improductivas. El análisis factorial realizado en la adaptación al castellano del instrumento obtuvo una solución similar a la de la versión original y la de otras adaptaciones (Chico et al., 2003), además se obtuvieron valores alfa de Cronbach de .78 para la subescala impulsividad funcional y de .76 para la subescala de impulsividad disfuncional. En la presente investigación los valores alfa de Cronbach fueron de .64 y .74 respectivamente, ligeramente más bajos que en el estudio de validación original pero aun así adecuados (Nunnally, 1967). Las dimensiones del cuestionario se relacionaron con la personalidad evaluada a través del instrumento EPQ-R (Aguilar, Tous, y Pueyo, 1990), en concreto la impulsividad funcional con la extraversión y la impulsividad disfuncional con el psicoticismo (Chico et al., 2003).

Evaluación del consumo de alcohol. Para evaluar el consumo de alcohol se empleó una única pregunta «Señala, en qué medida consumes alcohol» que se respondía en una escala Likert de cinco opciones de respuesta ( $1=$ nunca; $2=$ una o dos veces en el último año; $3=$ alguna vez al mes; $4=$ los fines de semana; $5=$ diariamente).

\section{Análisis de datos}

En un primer momento se analizaron los supuestos de linealidad, independencia (Durbin-Watson $=2.08$ ), normalidad y ausencia de multicolinealidad para todas las variables (niveles de tolerancia $>.1$ y Factor de Inflación de la Varianza, $F V I<10$ ), dividiendo en todos ellos 
la muestra según el género de los participantes. Para comprobar la existencia de diferencias en función del género en las variables del estudio, se empleó la prueba $t$ de Student y para las diferencias por curso académico se empleó ANOVA.

Para determinar si los estilos parentales o de crianza y la impulsividad de los adolescentes eran predictores de su consumo de alcohol se realizó un análisis de regresión múltiple por pasos, dividiendo la muestra según el género de los hijos, procediendo al análisis por separado para los hijos y para las hijas. Además de este análisis, para comprobar la estabilidad de los resultados de la regresión por pasos, se dividió cada submuestra (varones y mujeres) en dos submuestras seleccionadas aleatoriamente con el $50 \%$ de los participantes cada una y se repitieron los análisis.

Por último, para determinar si el estilo parental moderaba el efecto de la impulsividad del adolescente sobre el consumo de alcohol se realizó un análisis de moderación empleando el procedimiento bootstrap bias-corrected (Preacher y Hayes, 2008). Este método permite introducir tanto los efectos principales como los efectos de interacción simultáneamente y analizar el efecto del predictor (en este caso la impulsividad) a distintos niveles del moderador (en este caso baja, moderada y alta percepción de cada uno de los ocho dominios para padres y madres de estilos de crianza evaluados) que corresponden con la media y una desviación típica por encima y por debajo de la media (Preacher y Hayes, 2008).

\section{Resultados}

\section{Resultados descriptivos}

Respecto al consumo de alcohol el $26.3 \%$ de los participantes afirmó no consumir nunca alcohol, un 19.9\% manifestó haber consumido una o dos veces en el último año, el $23.6 \%$ alguna vez al mes, el $28 \%$ los fines de semana y un $2.2 \%$ tomaba alcohol diariamente.

La Tabla 1 muestra los valores descriptivos de las medidas empleadas, así como las diferencias en las mismas según el género de los participantes y el curso en el que se encontraban escolarizados. Se observaron diferencias por género en impulsividad disfuncional, con puntuaciones más altas para los varones. Respecto a los estilos de crianza, los chicos mostraban una puntuación significativamente más alta en evaluación negativa de la madre, negligencia tanto del padre como de la madre, y apoyo a la toma de decisiones del padre. En el caso de las chicas puntuaron más alto en sobreprotección materna. En relación al curso académico, no se observaron diferencias en impulsividad, pero sí en los estilos de crianza. Concretamente, hubo diferencias en todos los estilos de crianza maternos: conforme aumentaba la edad de los hijos (incremento en el curso académico) también lo hacía el apoyo a la toma de decisiones, evaluación positiva, permisividad, sobreprotección y desaprobación, mientras que disminuía la evaluación negativa, hostilidad y negligencia. Para los estilos de crianza

Tabla 1. Datos descriptivos de las medidas empleadas por género, curso y total

\begin{tabular}{|c|c|c|c|c|c|c|c|c|c|}
\hline & $\begin{array}{c}\text { Total } \\
(n=613) \\
M(D T)\end{array}$ & $\begin{array}{c}\text { Hombres } \\
(n=300) \\
M(D T)\end{array}$ & $\begin{array}{c}\text { Mujeres } \\
(n=313) \\
M(D T)\end{array}$ & $t$ & $\begin{array}{c}3^{\circ} \mathrm{ESO} \\
(n=142) \\
M(D T)\end{array}$ & $\begin{array}{c}4^{\circ} \mathrm{ESO} \\
(n=164) \\
M(D T)\end{array}$ & $\begin{array}{c}1^{\circ} \mathrm{BACH} \\
(n=169) \\
M(D T)\end{array}$ & $\begin{array}{c}2^{\circ} \mathrm{BACH} \\
(n=138) \\
M(D T)\end{array}$ & $F$ \\
\hline Impulsividad funcional & $5.64(1.73)$ & $5.90(1.38)$ & $5.37(1.65)$ & $3.872 * * *$ & $5.61(1.89)$ & $5.62(1.79)$ & $5.76(1.67)$ & $5.55(1.49)$ & .66 \\
\hline Impulsividad disfuncional & $6.01(2.11)$ & $5.96(2.16)$ & $6.05(2.06)$ & -.271 & $6.14(2.41)$ & $6.03(2.09)$ & $5.93(2.03)$ & $5.93(1.89)$ & .48 \\
\hline Madre. Apoyo decisiones & $21.06(4.03)$ & $20.73(3.97)$ & $21.36(4.07)$ & -1.95 & $21.48(3.82)$ & $20.36(4.27)$ & $20.48(4.21)$ & $22.16(3.42)$ & $6.29 * * *$ \\
\hline Madre. Evaluación negat. & $19.21(3.95)$ & $19.56(3.91)$ & $18.87(3.97)$ & $2.27^{*}$ & $19.89(4.10)$ & $19.31(4.19)$ & $19.33(3.46)$ & $18.19(3.94)$ & $4.54 * *$ \\
\hline Madre. Evaluación posit. & $12.83(2.31)$ & $12.66(2.29)$ & $12.99(2.33)$ & -1.95 & $12.90(2.28)$ & $12.30(2.31)$ & $12.65(2.36)$ & $13.65(2.07)$ & $8.36^{* * *}$ \\
\hline Madre. Permisividad & $9.01(1.51)$ & $8.95(1.70)$ & $9.06(1.31)$ & -1.05 & $8.90(1.55)$ & $8.97(1.57)$ & $8.80(1.32)$ & $9.42(1.54)$ & $4.51^{* *}$ \\
\hline Madre. Hostilidad-rigidez & $8.62(1.88)$ & $8.58(1.95)$ & $8.66(1.82)$ & -.38 & $9.04(1.96)$ & $8.54(1.93)$ & $8.51(1.72)$ & $8.40(1.91)$ & $3.86^{* *}$ \\
\hline Madre. Sobreprotección & $7.82(1.25)$ & $7.63(1.40)$ & $7.99(1.07)$ & $-3.67 * * *$ & $7.92(1.30)$ & $7.95(1.14)$ & $7.37(1.40)$ & $8.10(.95)$ & $9.97 * * *$ \\
\hline Madre. Negligencia & $5.04(1.63)$ & $5.18(1.59)$ & $4.90(1.65)$ & $2.158^{*}$ & $5.11(1.76)$ & $5.12(1.74)$ & $5.30(1.57)$ & $4.53(1.27)$ & $6.22 * * *$ \\
\hline Madre. Desaprobación & $6.21(1.33)$ & $6.11(1.40)$ & $6.31(1.25)$ & -1.37 & $6.10(1.45)$ & $6.22(1.37)$ & $6.04(1.27)$ & $6.54(1.16)$ & $3.19^{*}$ \\
\hline Padre. Apoyo decisiones & $28.38(5.48)$ & $28.92(5.09)$ & $27.87(5.78)$ & $2.08^{*}$ & $28.90(5.27)$ & $27.67(5.48)$ & $28.35(5.64)$ & $28.74(5.46)$ & 1.43 \\
\hline Padre. Evaluación negat. & $26.32(4.71)$ & $26.64(4.66)$ & $26.01(4.75)$ & 1.73 & $27.55(4.91)$ & $26.18(5.24)$ & $26.02(3.79)$ & $25.59(4.63)$ & $4.65^{* *}$ \\
\hline Padre. Sobreprot.-ignor. & $13.81(1.80)$ & $13.90(1.87)$ & $13.72(1.73)$ & 1.31 & $13.85(1.94)$ & $13.73(2.04)$ & $13.77(1.77)$ & $13.90(1.39)$ & .37 \\
\hline Padre. Negligencia & $5.14(1.62)$ & $5.29(1.57)$ & $4.98(1.66)$ & $2.22 *$ & $5.18(1.64)$ & $5.01(1.68)$ & $5.39(1.55)$ & $4.93(1.59)$ & 2.34 \\
\hline Padre. Permisividad & $7.24(1.65)$ & $7.35(1.68)$ & $7.13(1.63)$ & 1.36 & $6.91(1.69)$ & $7.11(1.58)$ & $7.49(1.61)$ & $7.44(1.70)$ & $3.76^{* *}$ \\
\hline Padre. Control & $3.47(1.10)$ & $3.55(1.15)$ & $3.40(1.05)$ & 1.47 & $3.58(1.21)$ & $3.45(1.06)$ & $3.62(1.08)$ & $3.22(1.03)$ & $4.60 * *$ \\
\hline Padre. Desaprobación & $4.12(1.15)$ & $4.14(1.15)$ & $4.10(1.15)$ & .57 & $3.99(1.21)$ & $4.22(1.19)$ & $4.12(1.05)$ & $4.13(1.15)$ & .78 \\
\hline Padre. Evaluación posit. & $3.93(1.12)$ & $3.92(1.07)$ & $3.93(1.16)$ & -.23 & $4.02(1.14)$ & $3.83(1.08)$ & $3.98(1.13)$ & $3.88(1.12)$ & .91 \\
\hline
\end{tabular}

Nota. ${ }^{*} p<.05,{ }^{* *} p<.01,{ }^{* * *} p<.001$ 
del padre se observaba un incremento de la permisividad y una disminución de la evaluación negativa y el control.

\section{Análisis de regresión}

En la Tabla 2 puede observarse tras el análisis de regresión que en el consumo de alcohol de los hijos varones influyen variables como el control del padre, la evaluación positiva de la madre y la desaprobación de la madre, explicando un $6.6 \%$ de la varianza. Las dos primeras vinculadas con la disminución del consumo de alcohol y la última, desaprobación de la madre, con el incremento en el consumo. Los análisis en las dos submuestras seleccionadas aleatoriamente en el grupo de valores arrojaron los mismos resultados, la varianza explicada era de hasta el 9.9\%.

En el caso de las hijas, las variables significativas fueron la evaluación positiva y la sobreprotección del padre y la impulsividad disfuncional, explicando en este caso, un $6.4 \%$ de la varianza en consumo de alcohol. En este caso la evaluación positiva del padre conlleva una disminución en el consumo de alcohol de sus hijas mientras que, por el contrario, la sobreprotección del padre y la impulsividad disfuncional se vincula con un incremento en el consumo. Al repetir los análisis con submuestras aleatorizadas se observaron los mismos resultados y una varianza explicada de hasta el 11.3\%.

Puesto que la impulsividad funcional no resultó significativa en los análisis de regresión anteriores, sólo se realizaron análisis de moderación utilizando la impulsividad disfuncional como factor en el modelo (categorizada como baja, media y alta en base a media y desviación típica), y las variables moderadoras analizadas fueron los estilos parentales paternos y maternos (clasificados como bajo, medio y alto uso en base a la media y desviación típica). Asimismo, los análisis se realizaron de manera independiente para hombres y mujeres de la muestra con objeto de adoptar una perspectiva de género.

En el caso de los varones, se observó que, de los ocho estilos de crianza para padres y madres analizados, dos estilos de crianza maternos y uno paterno moderaban el efecto que la impulsividad disfuncional del hijo tenía sobre el consumo de alcohol de este. Los estilos de crianza moderadores fueron los de evaluación negativa de la madre $(\beta=.018, D T=.009, t(298)=2.05, p<.05)$, negligencia de la madre $(\beta=.050, D T=.023, t(298)=2.13$, $p<.05)$ y control del padre $(\beta=.073, D T=.031, t(298)$ $=2.37, p<.05)$. El efecto de la evaluación negativa de la madre junto con la impulsividad disfuncional explicaban el consumo de alcohol de los hijos en un $2.3 \%\left(R^{2}=\right.$ $.023, F(3,296)=2.13, p<.05)$ y el efecto moderador de la evaluación negativa un cambio significativo en la varianza del consumo de alcohol de un $1.1 \%$ (cambio en $R^{2}$ $=.011, F(3,296)=2.99, p<.05)$. Respecto a la negligencia en la madre, su interacción con la impulsividad disfuncional de los hijos explicaba el consumo de alcohol en un $1.9 \%\left(R^{2}=.019, F(3,296)=2.77, p<.05\right) \mathrm{y}$ un incremento en la explicación del $1.7 \%$ por su efecto moderador (cambio en $R^{2}=.017, F(3,296)=4.57, p<$ $.05)$. Por último, el control del padre predecía el consumo de alcohol de los hijos en un $4.8 \%\left(R^{2}=.048, F(3\right.$, $296)=4.35, p<.01)$, y su efecto moderador un $2.1 \%$ adicional de varianza en el consumo (cambio en $R^{2}=$ $.021, F(3,296)=5.63, p<.05)$.

Como se puede observar en la Figura 1, si los hijos varones tenían puntuaciones altas en impulsividad disfuncional, su consumo de alcohol era mayor cuando la evaluación negativa (Figura 1A) o la negligencia de la madre (Figura 1B) eran altas. Sin embargo, cuando la impulsividad disfuncional de los hijos era baja, una alta evaluación negativa de sus madres se vinculaba con un menor consumo de alcohol.

En el caso de la estrategia parental control, una puntuación alta del padre se relacionaba con un incremento en el consumo de alcohol conforme aumentaba la impulsividad disfuncional de los hijos varones (ver Figura 1C). $\mathrm{Al}$ contrario, en varones con baja impulsividad disfuncional un mayor control se relaciona con el menor consumo.

Respecto a las mujeres, resultaron moderadores significativos del impacto de la impulsividad disfuncional sobre el consumo de alcohol, los estilos de crianza evaluación positiva del padre $(\beta=.054 ; D T=.024 ; t(311)=2.23, p<$

Tabla 2. Resultados de la regresión por pasos para el consumo de alcohol en varones y mujeres adolescentes

\begin{tabular}{|c|c|c|c|c|c|c|c|c|}
\hline Género & Paso & Variable & $B$ & $\beta$ & $R$ & $R^{2}$ & $R^{2}$ Corregida & Cambio en $R^{2}$ \\
\hline \multirow[t]{3}{*}{ Varón } & 1 & Control del padre & -.17 & $-.16^{*}$ & .160 & .025 & .021 & $.025^{*}$ \\
\hline & 2 & Desaprobación de la madre & .11 & $.13 *$ & .206 & .043 & .034 & $.017 *$ \\
\hline & 3 & Evaluación positiva de la madre & -.09 & $-.16^{*}$ & .256 & .066 & .053 & $.023 *$ \\
\hline \multirow[t]{3}{*}{ Mujer } & 1 & Evaluación positiva del padre & -.16 & $-.16^{*}$ & .162 & .026 & .022 & $.026 *$ \\
\hline & 2 & Sobreprotección-ignorancia del padre & .09 & $.14^{*}$ & .216 & .046 & .039 & $.020 *$ \\
\hline & 3 & Impulsividad disfuncional & .07 & $.13^{*}$ & .252 & .064 & .052 & $.017^{*}$ \\
\hline
\end{tabular}

Nota: $B$ : coeficientes no estandarizados, $\beta$ : coeficientes estandarizados. $* p<.05, * * p<.01$ 


\section{Hijos}
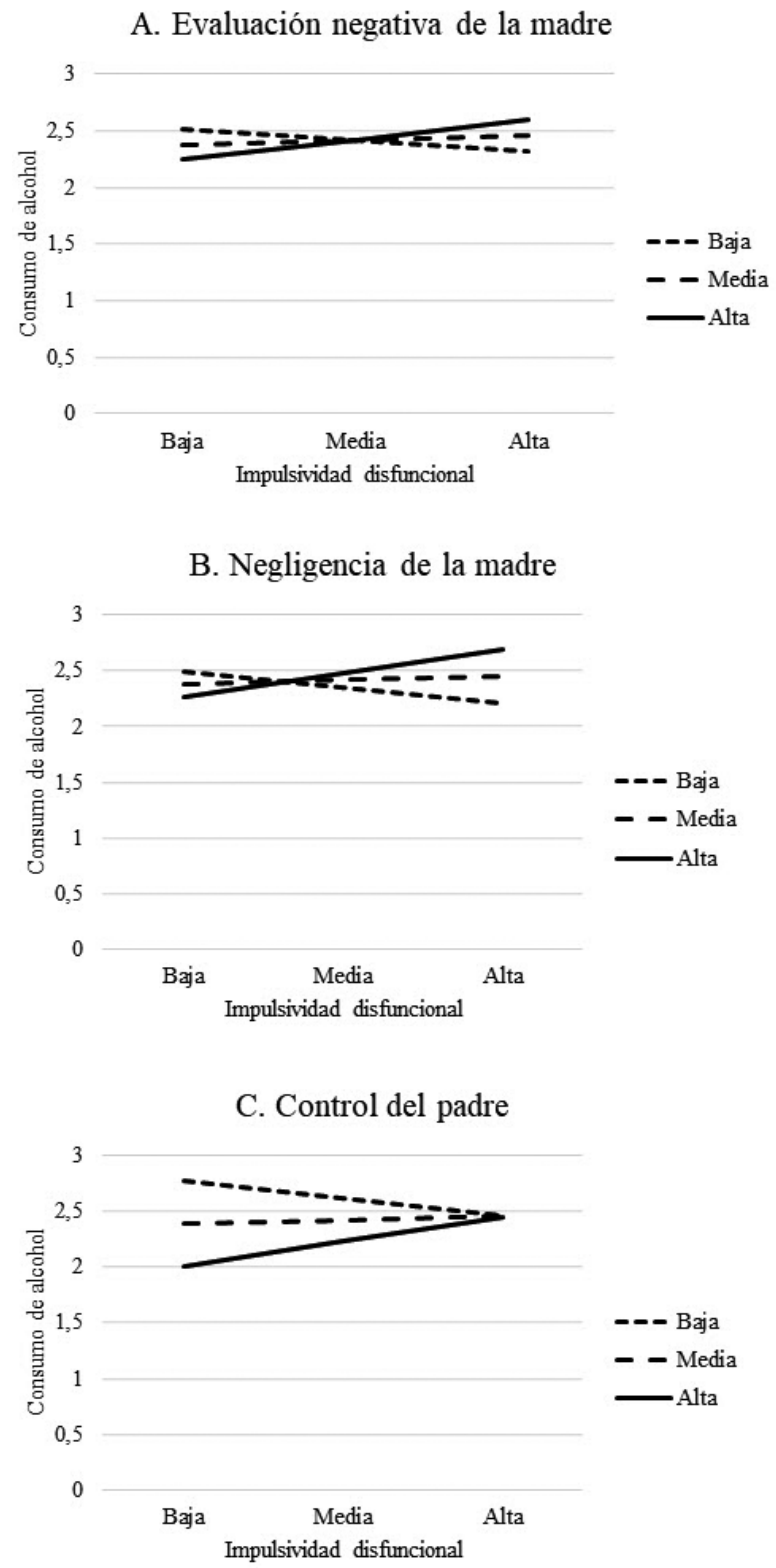

Hijas

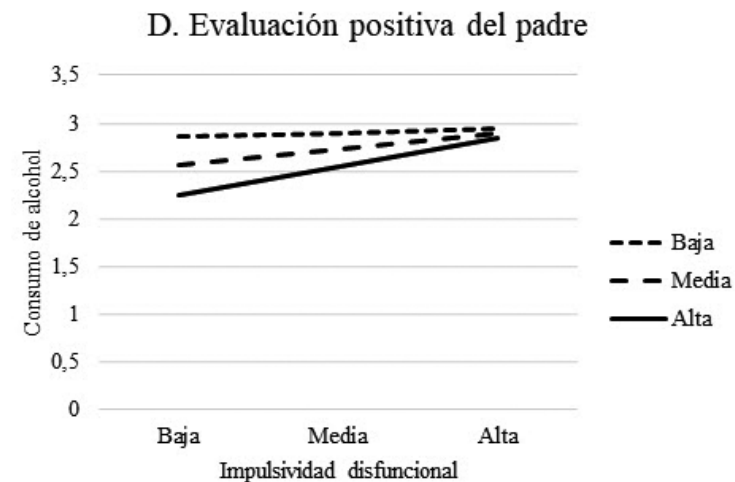

E. Apoyo y estimulación a la toma de decisiones del padre

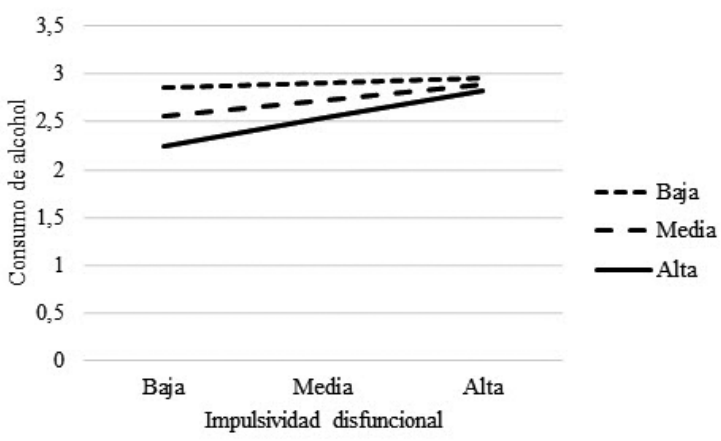

Figura 1. Efecto moderador de los estilos parentales maternos y paternos sobre la impulsividad disfuncional y el consumo de alcohol en sus hijos e hijas.

.05), y apoyo y estimulación a la toma de decisiones del padre $(\beta=.010 ; D T=.006 ; t(311)=1.80, p<.10)$.

La interacción de la impulsividad disfuncional de las hijas y la evaluación positiva del padre era capaz de explicar un $6.8 \%$ de la varianza en el consumo de alcohol de las hijas $\left(R^{2}=.068, F(3,309)=7.21, p<.01\right) \mathrm{y}$ el efecto moderador de la evaluación positiva del padre un $1.5 \%$ adicional (cambio en $R^{2}=.015, F(3,309)=4.98$, $p<.05)$. En la Figura 1 se puede observar que si la impulsividad de las hijas es baja se benefician de una alta evaluación positiva del padre (Figura 1D), de manera que eso hace que su consumo de alcohol sea menor. Si la impulsividad de las hijas es alta este estilo parental del padre no tiene efecto.

El otro estilo de crianza que moderaba, aunque marginalmente, la relación entre impulsividad y consumo de alcohol en las chicas era el apoyo y estimulación a la toma de decisiones por parte del padre. En este caso, la interacción explicaba un $6.2 \%$ de la varianza en el consumo de alcohol de las hijas $\left(R^{2}=.062, F(3,309)=\right.$ 
$6.21, p<.01)$ y el estilo de crianza del padre de apoyo y estimulación explicaba un $1.1 \%$ adicional en la varianza en consumo de alcohol (cambio en $R^{2}=.011, F(3,309)$ $=3.26, p<.10$ ). Este estilo parental del padre ejercía un efecto similar al de la evaluación positiva. Las hijas con baja impulsividad se benefician de un alto uso del control (apoyo y estimulación a la toma de decisiones) por parte del padre, pero cuando la impulsividad disfuncional en las hijas es alta no existe efecto (ver Figura 1E).

\section{Discusión}

En la actualidad, el consumo de alcohol en adolescentes es una seria preocupación tanto para las familias como para el sistema educativo, y la sociedad en general. En la muestra analizada en este trabajo, el $73.7 \%$ de los adolescentes manifiesta consumir alcohol, siendo esta cifra levemente inferior a la obtenida por un estudio reciente de ámbito nacional (ESTUDES, 2016), y dejando de manifiesto que prácticamente tres de cada cuatro adolescentes tienen algún tipo de relación con esta sustancia tóxica. Otro dato que resulta especialmente significativo es que el $2.2 \%$ de los estudiantes encuestados afirma beber a diario, cifra ligeramente superior a la obtenida en estudios previos con este colectivo, que se situaba en el 1.7\% (ESTUDES, 2016).

En este contexto, no es de extrañar el intenso debate científico existente sobre qué variables familiares pueden ejercer más peso en la prevención de este consumo. Diversas investigaciones han mostrado que las variables protectoras son el afecto, la comunicación positiva y el control mientras que la negligencia, la falta de apoyo y las pautas de crianza excesivamente coercitivas y reprobatorias aparecen vinculadas a un consumo mayor (Gázquez et al., 2016; Fuentes et al., 2015). Nuestros resultados van en la misma línea, mostrando la evaluación positiva y el control como variables protectoras generales clave del consumo de alcohol. No obstante, queremos destacar el papel mostrado en nuestro estudio por las verbalizaciones positivas, ya relacionadas anteriormente con la empatía y con el comportamiento prosocial (Mestre, Tur, Samper, Nácher, y Cortés, 2007), que parecen resultar tan importantes en la prevención del consumo como otras que tradicionalmente han tenido más peso, como es el caso de la variable control, que resulta más delicada ya que según cómo se emplee puede resultar beneficiosa o contraproducente en relación al consumo (Becoña et al., 2013).

Por otro lado, sabemos que los estilos de crianza pueden variar en función de las variables personales del adolescente haciendo más compleja la relación. Teniendo en cuenta la intensa relación entre la impulsividad y el consumo de alcohol que algunos investigadores consideran recíproca y bidireccional (Pilatti, Fernández, Viola, García, y Pautassi, 2017), hemos intentado profundizar en el papel que los estilos parentales pueden estar ejerciendo como factores moderadores en el binomio impulsividad -consumo de alcohol.

En nuestros resultados, los hijos varones con alta impulsividad disfuncional parecen beneficiarse de estilos de crianza maternos no negligentes y que no incluyan evaluaciones negativas. En concreto, les ayudará un entorno en el que la madre no utilice expresiones de ira y hostilidad, sino que use un estilo parental implicado y positivo (más comunicación, diálogo positivo, y que comparta actividades con ellos), y que no muestre descuido e ignorancia en relación a los comportamientos del hijo. De hecho, tradicionalmente se ha destacado en las investigaciones lo devastador que puede llegar a resultar un estilo negligente en la crianza y la importancia de proporcionar un ambiente en el que el adolescente se sienta seguro y confiado (Martínez-Loredo et al., 2016). Nuestros resultados señalan que esta implicación y aceptación de la familia cobra una especial relevancia cuando el adolescente presenta un alto grado de impulsividad.

También es de destacar la variable control por su papel diferencial en función de la presencia o no de impulsividad disfuncional en el hijo. De esta manera, solo los adolescentes varones con baja impulsividad se benefician de un estilo parental paterno caracterizado por un mayor control de su comportamiento. Por lo tanto, es una variable que va perdiendo importancia conforme aumenta el nivel de impulsividad del adolescente. En general, es probable que los chicos con poca impulsividad disfuncional posean más autocontrol, sean más dóciles, tranquilos, reflexivos y, en estos casos, un mayor grado de control parental ayude a ajustar su comportamiento y justifique su relación con un menor consumo de alcohol. En el caso de los chicos más impulsivos, parece menos adecuado utilizar sistemas tan controladores. Este dato puede contribuir al debate generado sobre la bondad de utilizar sistemas educativos con mayor o menor nivel de control. Asimismo, la investigación al respecto apunta que las estrategias parentales autoritarias o excesivamente coercitivas pueden incrementar la impulsividad del adolescente y favorecer el desarrollo de conductas desadaptadas. En cambio, los estilos basados en la aceptación pueden ayudar a controlar los impulsos (Jiménez-Barbero, Ruiz-Hernández, Velandrino-Nicolás, Llor-Esteban, y Waschgler, 2014). En el caso de las chicas, las que presentan un nivel bajo de impulsividad disfuncional se beneficiarán, en relación con el menor consumo de alcohol, de padres que hagan más evaluaciones positivas, empleen una comunicación con alto nivel de 
alabanzas y pocas críticas, y que les presten su apoyo y les estimulen en la toma de decisiones. En cambio, los estilos de crianza de la madre, en este caso, no se muestran como moduladores.

En esta investigación queda reflejada la importancia de distinguir las variables de género tanto de los hijos como de los progenitores, ya que los estilos parentales paternos y maternos no tienen el mismo efecto en los hijos y en las hijas adolescentes. De hecho, en nuestro estudio son significativas las variables de la madre, pero también cobran mucha importancia a estas edades las variables relacionadas con el padre. En general, aparece una relación moderadora más fuerte de los estilos de crianza maternos para los varones y de los paternos para las mujeres en el consumo de alcohol. En la literatura al respecto, también se observan diferencias según género de padres e hijos, pero los resultados son diversos. Para Perelló, Llorens, y Tortajada (2008) los aspectos educativos de la figura paterna tienen más peso en el consumo de alcohol. Sin embargo, en la investigación de Becoña et al. (2013) se observó que el afecto por parte de ambos padres y el control por parte de la madre, pero no del padre, fueron significativos en el consumo de drogas. En nuestro caso sí observamos que el control paterno tiene importancia en el caso de hijos con impulsividad disfuncional baja. Por lo tanto, es necesario profundizar en esta línea de investigación sobre los efectos diferenciales de los estilos educativos del padre y la madre como variables protectoras en el consumo de alcohol.

En cuanto a las limitaciones, este estudio presenta algunas reseñables. En primer lugar, resultaría de gran interés la utilización de un diseño longitudinal en lugar de uno transversal, ya que el primero es más apropiado para analizar las variables a largo plazo. Por otro lado, hemos de tener en cuenta también las restricciones de utilizar cuestionarios para recabar la información, ya que estamos valorando la percepción que tienen los adolescentes del estilo de crianza de sus padres, no el comportamiento de los padres en sí mismo. En este sentido, algunos investigadores han encontrado diferencias de género en la percepción de los diferentes estilos educativos, siendo los chicos más críticos en su manera de percibir los estilos familiares que las chicas (Mestre et al., 2010). Por ello sería interesante poder contar con la percepción de los propios padres sobre su manera de ejercer la parentalidad. Asimismo, realizar preguntas a los propios adolescentes sobre su consumo de alcohol puede originar un sesgo en las respuestas producido por la deseabilidad social. Esta limitación se ha intentado paliar garantizando el anonimato de las respuestas, pero sería más riguroso contar con un instrumento que evalúe esta variable.
Además, también sería importante analizar no solo si consumen alcohol sino también la cantidad de alcohol que consumen.

A pesar de estas limitaciones, los resultados reflejan el impacto de algunos estilos parentales como fuente de protección para la salud de los hijos adolescentes por su papel preventivo sobre el consumo de alcohol. También queda patente la importancia de diferenciar entre impulsividad funcional y disfuncional de los hijos, ya que es esta última la que se relaciona de forma significativa con el consumo de alcohol de los adolescentes de la muestra. En este sentido, encontramos, al igual que otros investigadores, que la efectividad de un estilo parental está vinculado a las características de los hijos (Grusec, Goodnow, y Kuczynski, 2000). De hecho, los comportamientos parentales que pueden beneficiar a los adolescentes más disfuncionalmente impulsivos no son generalizables a los no impulsivos.

En conclusión, conocer cómo el estilo parental puede actuar como moderador entre el comportamiento impulsivo y el consumo de alcohol puede facilitar el establecimiento de programas de prevención e intervención tanto sanitarios como educativos. Y es que, aunque estos programas destinados a incrementar la competencia de las familias hayan demostrado su eficacia (Gates, McCambridge, Smith, y Foxcroft, 2006), los educadores y sanitarios que participan en ellos deben conocer la vulnerabilidad al alcohol de aquellos adolescentes que tienen un perfil de personalidad más impulsivo, ya que son un grupo de riesgo, así como las variables familiares asociadas al consumo.

\section{Conflictos de intereses}

Los autores declaran que no existen conflictos de intereses.

\section{Referencias}

Aguilar, A., Tous, J. M., y Pueyo, A. (1990). Adaptación y estudio psicométrico del EPQ-r. Anuario de Psicología, 46, 101-118.

Alfonso, J. P., Huedo-Medina, T. B., y Espada, J. P. (2009). Factores de riesgo predictores del patrón de consumo de drogas durante la adolescencia. Anales de Psicología, 25, 330-338.

Ballester, R., y Gil, M. D. (2009). ¿Por qué los jóvenes se dan atracones de alcohol los fines de semana? Revista de Psicopatología y Psicología Clínica, 14, 25-35.

Baumrind, D. (1966). Effects of Authoritative Parental Control on Child Behavior, Child Development, 37, 887-907.

Becoña, E., Martínez, Ú., Calafat, A., Juan, M., Duch, M., y Fernández-Hermida, J. R. (2012). ¿Cómo influye la desorganización familiar en el consumo de drogas de los hijos? Una revisión. Adicciones, 24, 253-268. 
Becoña, E., Martínez, Ú., Calafat, A., Fernández-Hermida, J. R., Juan, M., Sumnall, H., Mendes, F., y Gabrhelík, R. (2013). Parental permissiveness, control, and affect and drug use among adolescents. Psicothema, 25, 292-298.

Campano, A., González-Tornaría, M. L., y Massonier, N. (2016). Estilos relacionales parentales: estudio con adolescentes y sus padres. Revista de Psicología, 34, 413-444.

Carballo, J. L., García G., Jaúregui, V., Marín, M., y Pérez-Jover, V. (2011). Análisis longitudinal de diferencias en habilidades cognitivas entre estudiantes de bachillerato consumidores de alcohol de la provincia de alicante. Health and Addictions, 11, 163-178.

Chico, E., Tous, J. M., Lorenzo-Seva, U., y Vigil-Colet, A. (2003). Spanish adaptation of Dickman's impulsivity inventory, its relationship to Eysenck's personality questionnaire. Personality and Individual Differences, 35, 1883-1892.

Collins, W. A., y Laursen, B. (2004). Parent-adolescent relationships and influences. En R. M. Lerner y L. Steinberg (Eds.), Handbook of Adolescent Psychology (pp. 331-361). Hoboken, NJ: Wiley.

Coloma-Medina, J. (1993). Estilos educativos paternos. En J. M. Quintana (Coord.), Pedagogía familiar (pp. 45-58). Madrid: Narcea.

Dickman, S. J. (1990). Functional and dysfunctional impulsivity: personality and cognitive correlates. Journal of Personality and Social Personality, 58, 95-102.

Echeburúa, E., y Corral, P. (2010). Adicción a las nuevas tecnologías y a las redes sociales en jóvenes: un nuevo reto. Adicciones, 22, 91-96.

Fuentes, M. C., Alarcón, A., Gracia, E., y García, F. (2015). El ajuste escolar en los adolescentes españoles: influencia de la socialización parental. Cultura y Educación, 27, 1-32.

García-Moreno, L., Expósito, J., Sanhueza, C., y Ángulo, M. (2008). Actividad prefrontal y alcoholismo de fin de semana en jóvenes. Adicciones, 20, 271-280.

Gates, S., McCambridge, J., Smith, L. A., y Foxcroft, D. R. (2006) Interventions for prevention of drug use by young people delivered in non-school settings. The Cochrane Database of Systematic Reviews, 1, CD005030.

Gázquez, J. J., Pérez-Fuentes, M. C., Molero, M. M., Barragán, A. B., Martos, Á., y Sánchez-Marchán, C. (2016). Drug use in adolescents in relation to social support and reactive and proactive aggressive behavior. Psicothema, 28, 318-322.

Golpe, S., Isorna, M., Barreiro, C., Braña, T., y Rial, A. (2017). Consumo intensivo de alcohol en adolescentes: prevalencia, conductas de riesgo y variables asociadas. Adicciones, 29, 256-267.

Grusec, J. E., Goodnow, J. J., y Kuczynski, L. (2000). New directions in analyses of parenting contributions to children's acquisition of values. Child Development, 71, 205-211.

Hernández-López, T., Roldán, J., Jiménez, A., Mora, C., Escarpa, D., y Pérez-Álvarez, M. T. (2009). La edad de inicio en el consumo de drogas, un indicador de consumo problemático. Psychosocial Intervention, 18, 199-212.
Hernando, A., Oliva, A., y Pertegal, M. A. (2012). Variables familiares y rendimiento académico en la adolescencia. Estudios de Psicología, 33, 51-65.

Iglesias, B., y Romero, E. (2009). Estilos parentales percibidos, psicopatología y personalidad en la adolescencia. Revista de Psicopatología y Psicología Clínica, 14, 63-77.

Jiménez-Barbero, J. A., Ruiz-Hernández, J. A., Velandrino-Nicolás, A. P., y Llor-Zaragoza, L. (2016). Actitudes hacia la violencia, impulsividad, estilos parentales y conducta externalizada en adolescentes: comparación entre una muestra de población general y una muestra clínica. Anales de Psicología, 32, 132-138.

Kenney, S. R., Lac, A., Hummer, J. F., Grimaldi, E. M., y LaBrie, J. W. (2015). Pathways of Parenting Style on Adolescents' College Adjustment, Academic Achievement, and Alcohol Risk. Journal of College Student Retention: Research, Theory \& Practice, 17, 186-203.

López-Caneda, E., Mota, N., Crego, A., Velásquez, T., Corral, M., Rodríguez Holguín, S., y Cadaveira, F. (2014). Anomalías neurocognitivas asociadas al consumo intensivo de alcohol (binge drinking) en jóvenes y adolescentes: Una revisión. Adicciones, 26, 334-359.

Luque, L., Gómez, R., Cortés, M. T., Espejo, B., y Giménez, J. A. (2014). Consumo de alcohol en atracón en jóvenes. Evaluación de un instrumento basado en la teoría de la conducta planificada. Revista Argentina de Ciencias del Comportamiento, 6, 65-74.

Maccoby, E. E., y Martin, J. A. (1983). Socialization in the context of the family: Parent-child interaction. En P. H. Mussen (Ed.), Handbook of child psychology (Vol. 4, pp. 1-101). New York: Wiley.

Martínez, I., Fuentes, M., García, F., y Madrid, I. (2013). El estilo de socialización familiar como factor de prevención o riesgo para el consumo de sustancias y otros problemas de conducta en los adolescentes españoles. Adicciones, 25, 235-242.

Martínez-Loredo, V., Fernández-Artamendi, S., Weidberg, S., Pericot, I., López-Núñez, C., Fernández-Hermida, J. R., y Secades, R. (2016). Parenting styles and alcohol use among adolescents: A longitudinal study. European Journal of Investigation in Health, Psychology and Education, 6, 27-36.

Mestre, M. V., Tur, A. M., Samper, P., Nácher, M. J., y Cortés, M. T. (2007). Estilos de crianza en la adolescencia y su relación con el comportamiento prosocial. Revista Latinoamericana de Psicología, 39, 211-225.

Narváez, D. A., y Caro, E.J. (2015). Impulsividad funcional y disfuncional en adolescentes consumidores de alcohol. Revista Electrónica de Psicología Iztacala, 18, 539-563.

Nunnally, J. C. (1967). Psychometric Theory (1st ed.). New York: MacGraw-Hill.

Observatorio Español de la droga y las toxicomanías. (2015). Informe 2015. Alcohol, tabaco y drogas ilegales en España. Madrid: Ministerio de Sanidad. Recuperado de http://www.pnsd. msssi.gob.es/profesionales/publicaciones/catalogo/catalogoPNSD/publicaciones/pdf/INFORME_2015.pdf

Oliva, A., y Antolín, L. (2010). Cambios en el cerebro adolescente y conductas agresivas y de asunción de riesgos. Estudios de Psicología, 31, 53-66.

Orozco-Cabal, L. F., y Herin, D. (2008). Neurobiología de la impulsividad y los trastornos de la conducta alimentaria. Revista Colombiana de Psiquiatría, 37, 207-219. 
Pedrero, E., y Ruiz, J. M. (2011). Factores de vulnerabilidad para desarrollar una adicción: elementos para su prevención. En E. Pedrero (Coord.), Neurociencia y adicción (pp. 99-110). Madrid: Sociedad Española de Toxicomanías y Plan Nacional sobre Drogas.

Pedrero, E., Ruiz, J. M., Rojo, G., Llanero, M., y Puerta, C. (2012). Caracterización neuropsicológica de la impulsividad funcional y disfuncional en adictos a sustancias: implicaciones clínicas. Adicciones, 24, 51-58.

Perelló, M. J., Llorens, N., y Tortajada, S. (2008). Influencia de los estilos educativos paternos en el consumo de drogas en adolescentes. Revista Española de Drogodependencias, 33, 288-299.

Petit, G., Maurage, P., Kornreich, C., Verbanck, P., y Campanella, S. (2014). Binge drinking in adolescents: A review of neurophysiological and neuroimaging research. Alcohol and Alcoholism, 49, 193-197.

Pérez Alonso-Geta, P. M. (2012). La socialización parental en padres españoles con hijos de 6 a 14 años. Psicothema, 24, 371-376.

Pilatti, A., Fernández, C., Viola, A., García, J. S., y Pautassi. R .M. (2017). Efecto recíproco de impulsividad y consumo de alcohol en adolescentes argentinos. Health and Addictions, 17, $107-120$
Plan Nacional sobre Drogas. (2016). Informe ESTUDES 2014-15. Madrid: Ministerio de Sanidad y Consumo. Recuperado de: http://www.pnsd.msssi.gob.es/profesionales/sistemasInfor$\mathrm{mac}$ ion/sistemaIn for macion/p f f/2016_ES T U DES_2014-2015.pdf

Preacher, K., y Hayes, A. (2008). Asymptotic and resampling strategies for assessing and comparing indirect effects in multiple mediator models. New York, NY: Springer.

Pons, J. y Berjano, E. (1997). Análisis de los estilos parentales de socialización asociados al abuso de alcohol en adolescentes. Psicothema, 9, 609-617.

Raya, A. F., Herruzo, J., y Pino, M. J. (2008). El estilo de crianza parental y su relación con la hiperactividad. Psicothema, 20, 691-696.

Samper, P., Cortés, M. T., Mestre, V., Nácher, M. J., y Tur, A. M. (2006). Adaptación del Child's Report of Parent Behavior Inventory a población española. Psicothema, 18, 263-271.

Schaefer, E. S. (1965). Children's reports of parental behavior: an inventory. Child Development, 36, 414-424.

Tur, A. M., Mestre, V., Samper, P., y Malonda, E. (2012). Crianza y agresividad de los menores: ¿es diferente la influencia del padre y de la madre? Psicothema, 24, 284-288. 
